\title{
The paradigm of the area law and the structure of transversal and longitudinal lightfront degrees of freedom
}

\author{
Bert Schroer \\ present address: CBPF, Rua Dr. Xavier Sigaud 150 \\ 22290-180 Rio de Janeiro - RJ, Brazil \\ email Schroer@cbpf.br \\ Prof. emer. of the FU-Berlin
}

May 2002

\begin{abstract}
It is shown that an algebraically defined holographic projection of a QFT onto the lightfront changes the local quantum properties in a very drastic way. The expected ubiquitous vacuum polarization characteristic of QFT is confined to the lightray (longitudinal) direction, whereas operators whose localization is transversely separated are completely free of vacuum correlations. This unexpected "transverse return to QM" combined with the rather universal nature of the strongly longitudinal correlated vacuum correlations (which turn out to be described by rather kinematical chiral theories) leads to a d-2 dimensional area structure of the d-1 dimensional lightfront theory. An additive transcription in terms of an appropriately defined entropy related to the vacuum restricted to the horizon is proposed and its model independent universality aspects which permit its interpretation as a quantum candidate for Bekenstein's area law are discussed. The transverse tensor product foliation structure of lightfront degrees of freedom is essential for the simplifying aspects of the algebraic lightcone holography.
\end{abstract}

Key-words: Quantum field theory; Mathematical physics, Quantum gravity

\section{Introduction}

Peculiarities of lightfront and " $p \rightarrow \infty$ frame" behavior in particle physics have been noticed in many publications starting from the beginnings of the 70s [1]. In recent times we have seen a renewed interest in the subject as a result of the idea of holography [2] i.e. the conjecture that for certain geometric constellations it may be possible to encode degrees of freedoms and most of the properties of a QFT in d-spacetime dimensions into a suitably chosen lower dimensional geometric carrier. Although the first intuitive picture about such encoding came from consistency observations on rotational symmetric black holes, there were also arguments that a similar holographic encoding may occur in plane Minkowski space lightfront physics [3]; in fact such an idea receives additional support from the analogy of the Hawking effect caused by black holes with bifurcated horizons with the Unruh effect associated with the Rindler wedge in Minkowski spacetime [4] in the sense that if there exists an analogy on the thermal side of a Hawking temperature and that seen by an Unruh observer, there should also be an analogous behavior of other thermodynamic aspects as e.g. a suitably defined "localization entropy" associated to the horizon (i.e. half of the lightfront). Since a thermal interpretation in terms of fundamental laws imposed on the black hole situation requires the Bekenstein area law for entropy, and since the finite surface of a black hole corresponds to the infinite surface defined by the edge of the Rindler wedge, one would expect to find a rather universal constant surface density of the entropy of the vacuum which in its restriction to a suitably defined subalgebras on the (upper) horizon of the Rindler wedge is described by a density matrices.

The main aim of this note is to present new concepts and mathematical tools which show that this is not only an analogy between special situations in curved spacetime and some new aspects of lightfront 
physics (as compared to the way the lightfront and the $p \rightarrow \infty$ frame method were previously used in particle physics), but rather the start of a paradigmatic change in looking at local quantum physics 1 . In order to appreciate this statement the reader is reminded that for several decades there exist two ways of dealing with QFT which are mainly different in their interpretation, mathematical implementation of concepts and underlying philosophy but which were based on a shared stock of principles. The standard approach (i.e. that of most textbooks and used by the vast majority of researchers) attributes a direct physical reality to pointlike quantum (suitably averaged in the sense of Bohr and Rosenfeld) fields, whereas the operator-algebraic setting is based on the idea that joint properties of algebras generated by operators which share the same spacetime localization already contain all the physics (analogous to the fundamental role of localization of events in with particle counters whose inner working is in most cases unknown [10]), but in order to unravel it one cannot use the standard methods.

The historically first observation which lend some respectability to the algebraic viewpoint is the natural explanation of the insensitivity of the S-matrix against local changes of the field-coordinatization. The support from the S-matrix viewpoint was strengthened by the recent observation that unitary crossing symmetric (this being the on-shell substitute for the missing off-shell Einstein causality) have maximally one system of local algebras if they have one at all [11]. However in many cases these observations which deemphasize the role of individual fields in favour of local equivalence classes really do not require the use of the algebraic viewpoint since e.g. the S-matrix continues to be expressible by LSZ formulas in terms of pointlike fields. This is quite different in the present case of lightfront holography [34] [5].

Although we are aware that the general public level of knowledge about modular theory, on which this paper is based, is lagging far behind its importance in local quantum physics, this short note is not the place for looking at background information. For attempts in this direction and comments on its interesting history we rather refer to relevant sections in [10] and 12 .

The present algebraic lightfront holography incorporates concepts which have attracted attention in various areas of particle physics, curved spacetime QFT [- 1 , basic quantum physics and quantum measurement [14] and even in physics-based philosophical contributions [13].

For the convenience of the reader we have collected the previously presented results on lightfront restrictions of free fields as well as some geometric definitions concerning lightfront, wedges and their lightfront-horizon [5] 34 in compressed form in an appendix. The reader is strongly advised to have a look at the results obtained by this mathematically more elementary lightfront restriction method (which is limited to free fields) before he reads the next section.

\section{The setting of local quantum physics and its adaptation to the lightfront}

In the derivation of the time-dependent scattering theory and the analytic properties carried out in the $50 \mathrm{~s}$ and $60 \mathrm{~s}$ it became clear that pointlike fields are analogous to coordinates in differential geometry. These investigations also increased the awareness about the limitations of the adaptation of the classical field picture to local quantum physics ("quantization") and, in particular, suggested that the attribution of a physical reality to individual fields may be a bit of an illusion which in certain cases may even conceal basic intrinsic properties. This is indeed the case for QFT on the lightfront as we hope to make clear in the sequel.

It is assumed that the reader knows some basic facts about the algebraic approach which describes QFT in terms of a collection (net) of spacetime indexed operator algebras 10] in a common Hilbert space fulfilling causality- covariance- and spectral- properties. These properties are adaptations of those which already appeared in the first non-Lagrangian setting of QFT by Wightman [15 and which nowadays are often referred to as the linear Wightman requirements. Besides the linear Einstein causality (spacelike local commutativity) there are also causality requirements which are an algebraic substitute for an equation of motion namely the causal shadow property or primitive causality 16

$$
\mathcal{A}(\mathcal{O})=\mathcal{A}\left(\mathcal{O}^{\prime \prime}\right)
$$

\footnotetext{
${ }^{1}$ There are indications for an ongoing paradigmatic change in various other areas of QFT [6] [6] 8 [9].
} 
i.e. the operator algebra (always weakly closed) localized in a simply connected region $\mathcal{O}$ equals that of its causal completion $\left(\mathcal{O}^{\prime \prime}\right.$ is the spacelike disjoint of $\mathcal{O}^{\prime}$ which in turn is the spacelike disjoint of $\left.\mathcal{O}\right)$. Such properties have no natural expressions in terms of the mainly linear Wightman properties and rather require the setting of operator algebras [16]. Since we will also be concerned with subalgebras associated with lower-dimensional regions as, e.g., lightfront algebra $\mathcal{A}(L F)$ we will also assume the characteristic extension of causal shadow properties of which the following relation is the most important case

$$
\mathcal{A}(W)=\mathcal{A}\left(L F_{+}\right)
$$

Classically its content and validity should be obvious: the field data in the (Rindler) wedge $W=$ $\left\{x|| x^{0} \mid<x^{1}, x^{1}>0\right\}$ are determined in terms of the characteristic data on its upper causal horizon $L F_{+}$(the $x^{1}>0$ half of the light front). $W$ is the causal shadow of $L F_{+}$since every particle or lightray which has passed through $L F_{+}$must have before passed through $W$ and vice versa. But note that a region on $L F$ which is bounded in the $x^{1}$ direction does not cast any causal shadow at all; this is one of the peculiarities of the lightfront. In QFT it has been shown for free fields [20] [21] and holds (within the present level of mathematical rigor [3] in the algebraic formulation of $d=1+1$ factorizing models [24]. Since it is natural extension of the causal shadow principle (limiting case of the spacelike causal shadow principle of local quantum physics [10]) and fits perfectly into the modular setting, we add it to the list of requirements of local quantum physics leaving open the very plausible possibility that it may turn out to follow already from the commonly accepted principles. The only exception for the validity of (2) are $\mathrm{d}=1+1$ chiral theories.

The operator algebra on the left hand side in (2) is defined in terms of the net-setting of AQFT as all operator algebras associated with noncompact localization regions namely

$$
\mathcal{A}(W)=\overline{\bigcup_{\mathcal{O} \subset W} A(\mathcal{O})}
$$

where the closure is in the weak operator topology. Such observable algebras are required to obey the geometric Bisognano-Wichmann property $\mathcal{A}\left(W^{\prime}\right)=\mathcal{A}(W)^{\prime}$ where $W^{\prime}$ is the geometric opposite (spacelike disjoint) of $W$ and the dash on the operator algebra denotes as usual the von Neumann commutant algebra 10. Wedge algebras are actually von Neumann factors i.e. $\mathcal{A}(W) \cap \mathcal{A}(W)^{\prime}=\mathbb{C} \mathbf{1}, \mathcal{A}(W) \vee \mathcal{A}(W)^{\prime}=B(H)$ 12. The upper horizon algebra $\mathcal{A}\left(L F_{+}\right)$on the right hand side is best defined in terms of the result of the following theorem on modular inclusion:

Theorem 1 (Wiesbrock, Borchers [17] 192) Let $W_{e_{+}} \subset W$ be the lightlike translated wedge algebra $e_{+}=(1,1,0,0)$. The inclusion of operator algebras

$$
\mathcal{A}\left(W_{e_{+}}\right) \equiv A d U\left(e_{+}\right) \mathcal{A}(W) \subset \mathcal{A}(W)
$$

is "modular" i.e. the modular unitary $\Delta_{W}^{i t}$ of the standard pair $\left(\mathcal{A}\left(W_{e_{+}}\right), \Omega\right)$ "compresses" the smaller algebra

$$
\sigma_{t, W}\left(\mathcal{A}\left(W_{e_{+}}\right)\right) \equiv A d \Delta_{W}^{i t} \mathcal{A}\left(W_{e_{+}}\right) \subset \mathcal{A}\left(W_{e_{+}}\right), t>0
$$

In this case the original positive energy lightray translation $U_{e_{+}}(a) \equiv U\left(a e_{+}\right)$can be recovered from the two modular unitary groups $\Delta_{W}^{i t}, \Delta_{W_{e_{+}}}^{i t}$, and the lightlike translation $U_{e_{+}}(a)$ together with the modular group $\Delta_{W}^{i t}$. obey the Borchers translation-dilation commutation relation

$$
\Delta_{W}^{i t} U_{e_{+}}(a)=U_{e_{+}}\left(e^{-2 \pi t} a\right) \Delta_{W}^{i t}
$$

If the relative commutant

$$
\mathcal{A}\left(W_{e_{+}}\right)^{\prime} \cap \mathcal{A}(W)
$$

\footnotetext{
${ }^{2}$ Our reference wedge will be always the $x^{0}-x^{1}$-wedge, the wedge in any other position is obtained by applying Poincaré transformations.

${ }^{3}$ The present constructive methods for $\mathrm{d}=1+1$ factorizable models deal with bilinear forms (matrix elements of "would be" operators) and fall still short of genuine operator algebra methods.
} 
is also standard with respect to the vacuum $\Omega$ (in which case the modular inclusion is called "standard'用), then the two definitions

$$
\begin{aligned}
\mathcal{A}(L F(0,1)) & \equiv \mathcal{A}\left(W_{e_{+}}\right)^{\prime} \cap \mathcal{A}(W) \\
\mathcal{A}\left(L F_{+}\right) & \equiv \bigcup_{a>0} A d U_{e_{+}}(a) \mathcal{A}(L F(0,1)) \\
\mathcal{A}\left(L F_{-}\right) & =J \mathcal{A}\left(L F_{+}\right) J
\end{aligned}
$$

lead to

$$
\begin{aligned}
& \mathcal{A}\left(L F_{+}\right)=\mathcal{A}(W) \\
& \mathcal{A}\left(L F_{-}\right)=\mathcal{A}(W)^{\prime}=\mathcal{A}\left(W^{\prime}\right)
\end{aligned}
$$

Here $J$ is the modular involution of the standard pair $(\mathcal{A}(W), \Omega)$. The resulting net structure on the lightfront algebra $\mathcal{A}(L F)$ is that of a generalized chiral theory; in addition to invariance under lightray translation and dilations ( (6) the vacuum is invariant under a positive generator $L_{0}$ rotation [18] whose action on the original net is "fuzzy" [4] (not representable by a diffeomorphism).

This theorem requires several comments. In its present form it is the adaptation of an abstract mathematical theorem on modular inclusion to local quantum physics. In fact it comprises three closely intertwined theorems, the related Borchers- and Wiesbrock- theorems [12] and a theorem on the equivalence of standard modular inclusions with generalized chiral QFTs $[18]$.

The standard modular inclusion theorem resolves the problem of the longitudinal localization structure of the lightfront algebra, but it is "blind" with respect to the transverse direction of the edge of the bifurcated horizon of a Rindler wedge. It is precisely the presence of these transverse degrees of freedom 5 which distinguish the generalized chiral theories of the previous theorem from those more familiar standard chiral theories which result from the chiral tensor decomposition of $\mathrm{d}=1+1$ conformal theories. The former permit the nontrivial action (transverse translations and rotation) of automorphisms which, in lack of a transverse localization structure, are at first sight hard to distinguish from internal symmetries. However, in the next section, we will show how to construct a transverse localization with quite unexpected properties (alluded to in the abstract) using the methods of AQFT. The relevant Poincaré transformations are those which appeared before in the attempts to reconstruct the original massive net from its holographic lightcone projection .

Let us collect those properties in form of a condition on which our analysis relies but which were not part of Wightman's framework [15] of what constitutes a QFT.

Condition 2 (extended causal shadow property and cyclicity condition) Physically admissable models of local quantum physics fulfill in addition to the standard linear properties (local commutativity, covariance and spectral positivity) also the causal shadow property including its extension (国) as well as the cyclicity (Reeh-Schlieder property) of all relative commutants (even if they the localization region, as it happens e.g. for compact regions on the lightfront, do not cast a causal shadow i.e. are identical to their causal completion) as used in the previous modular inclusion theorem.

These causality properties allow to geometrically identify (even arbitrarily tight-localized) subalgebras which fulfill the Reeh-Schlieder cyclicity; they turn out to be essential for the formulation of lightfront holography. Whereas in quantum mechanics (say in the multiplicative form of second quantization in order to facilitate the comparison with QFT) a quantization box splits the world into a tensor product

\footnotetext{
${ }^{4}$ The terminology "standard" refers to a pair (algebra, reference state vector $)=(\mathcal{A}, \Omega)$ and stands for the cyclicity $(\overline{\mathcal{A} \otimes}=$ $H)$ and the separating property $(A \Omega=0, A \in \mathcal{A} \curvearrowright A=0)$. In QFT the reference state is usually the vacuum vector and the standardness is called the Reeh-Schlieder property (or somewhat imprecisely "the operator-statevector relation").

${ }^{5}$ The "degree of freedom" terminology and their counting in the calculation entropy makes literal sense only for free (or systems with quanta similar to free systems) quantum field systems; its meaning outside is of a more physical intuitive nature, which receives some support from the phase space structure of local quantum physics 10 ].

${ }^{6}$ This condition does not contradict the well-known limitation of the canonical equal time formalism since the double-cone algebras (which become associated with an equal time base via this causal shadow property) admit generally no generating equal time fields. The situation on the lightfront is totally different.
} 
between the inside and outside quantum mechanical Fock spaces in such a way that the vacuum factorizes without entanglement, the QFT situation is the extreme opposite: from the algebra of an arbitrary small spacetime region (e.g. a small double cone as the prototype of a relativistic box) one can approximate any state of the world from the vacuum. The denseness of the subspace cyclically generated from the vacuum is always guarantied as long as the open localization region has a nontrivial causal disjoint. Accepting this condition as a characterization of "physically admissable" is a reasonable working hypotheses (all the QFT which have been used fall into this category) and should not be confused with installing a doctrine. Stringlike QFTs as Wigner's "helicity towers" (QFTs associated with zero mass faithful little group representations which contain all helicities including the helicity of the graviton $h=2$ ) which do not permit a nontrivial localization in compact regions are of course legitimate objects of further research. This is especially so since their quantum consisteny requires the semiinfinite stringlike localization in any spacetime dimensions (more precisely the spacelike cone localization of algebraic QFT), but does not bring about restrictions to $\mathrm{d}=10,24$ spacetime dimensions. The reason for the disharmony between the causality and stability principles of QFT and string theory is not that string theory invents structures which are new and untested but rather that its premature and unfounded claim of being a theory of everything precludes the necessary work to confront the principles of QFT and in this way reveal (as it was done with all physically relevant new theories before) its possible new aspects.

A very important mathematical tool in the ongoing paradigmatic change of QFT is the TomitaTakesaki modular theory of operator algebras. All the significant differences to QM may be traced back to the phenomenon of vacuum polarization which in turn is a direct consequence of the causality and spectral stability properties (positivity of timelike translation generators). The omnipresence of vacuum polarizations and the related spacelike correlations in vacuum expectations of products of spacelike separated observables even in interaction-free theories is a well-known phenomenon ever since Heisenberg discovered it through studying the global limit of local Noether charges in free field theories in 1934 [19].

A closely related refinement of the manifestation of vacuum polarization which is able to detect the presence of interactions (and therefore is of direct relevance to particle physics) is expressed in the following recent theorem on existence of properties of "polarization-free generators" (PFG) of localized one-particle creation operators. A PFG $G$ is an (generally unbounded) operator affiliated with an operator algebra $\mathcal{A}(\mathcal{O})$ (in mathematical notation) $G \eta \mathcal{A}(\mathcal{O})$ such that (\# denotes as usual either no superscript or the hermitian conjugation)

$$
G^{\#} \Omega=1-\text { particle vector }
$$

Theorem 3 (297) Wedge-localized operator algebras in theories describing massive particles always possess affiliated PFGs F

$$
G \eta \mathcal{A}(W)
$$

whereas the existence of PFGs for algebras localized in causally closed subwedge regions imply the absence of interactions in the subspaces (sectors) generated by repeated application of $G$ to the vacuum; in fact in this case the translates of PFGs $G(x)=A d U(x) G$ are actually linearly related to free fields.

This theorem is a strengthened form within the operator algebra setting of an old well-known theorem which characterizes free fields in terms of two-point functions [15] and which recently among other things turned out to be of interest in connection with the protection mechanism in conformal SYM theories [23].

These aspects of PFGs are interesting for two reasons. On the one hand one obtains an entirely intrinsic characterization of absence/presence of interactions which does not depend on the chosen field coordinatization in the equivalence class of all local fields 7 . On the other hand the special role attributed to wedge algebras may be used as the start of a new constructive approach to QFT. Although the interaction is at first sight not directly visible on the level of the wedge algebra generated by wedgeaffiliated PFGs, it makes its appearance in the properties of the modular operators 24]. The TCP-related modular involution $J$ turns out to differ from its interaction-free form by the appearance of the scattering operator $S_{\text {scat }}$, which thereby acquires a new role related to localization (which completely escaped the old S-matrix philosophy according to which on-shell quantities do not reveal localization properties). Although it has up to now not been possible to show the existence of a QFT associated with a given

\footnotetext{
${ }^{7}$ Since the Wick-polynomials of free fields have quite complicated correlation functions, it would be difficult to assert the absence of interactions by just looking at them.
} 
admissable (unitary, crossing symmetric with the necessary analytic properties) S-matrix, the application of modular theory does lead to the uniqueness of the associated would be AQFT, i.e. there is either none or just one local quantum theory with an admissable S-matrix [11. Knowing the structure of the wedge algebra one may construct the operator algebras of smaller regions by forming algebraic intersections (instead of restricting the support of smearing functions as one would restrict localization regions in the standard approach). That this completely intrinsic algebraic way is practicable has been shown recently in some "well behaved" but nevertheless nontrivial two-dimensional models of factorizable models. The mysterious nonlocal Zamolodchikov-Faddeev algebra which appeared as a computational tool acquires for the first time a spacetime interpretation: its Fourier transforms are the PFGs of the wedge algebra 24.

\section{Tranverse Localization on the Lightfront and the return of vacuum-polarization-free Quantum Mechanics}

We now turn to the issue of equipping the transverse lightfront directions with a localization structure \$. $^{\text {f }}$ The modular inclusion techniques of the previous section has provided us with a chiral net. But it would be wrong to think of these horizon-affiliated chiral nets as being of the same kind as those families which one has found by chiral tensor decomposition of $\mathrm{d}=1+1$ dimensional conformal theories. Even though modular theory suggests strongly that any Moebius-covariant chiral net contains a representation of the diffeomorphism group of the circle (but without the possibility of defining a physical energy-momentum tensor through the Witt-Virasoro infinitesimal generators $L_{n}$ ), there is absolutely no reason to expect the existence of a finite trace (character) of the Gibbs operator $e^{-\beta L_{0}}$ since the transverse translational symmetry would force each potential eigenvalue to be infinitely degenerate. With other words one is dealing with a generalized chiral situation in which one cannot expect the important "split property" 10] to hold9 even if it was valid in the original d-dimensional ambient theory. This transverse alignment of degrees of freedom is just another illustration of the rather radical aspects of algebraic holography which are the prize to be paid for the gain in kinematical simplicity and universality of certain algebraic structures. It is the main reason why measures of the relative cardinality of quantum field theoretical situations in which the thermal aspects are caused by localization exhibit an an area law instead of the volume proportionality of entropy associated with standard heat bath thermal physics.

Hence the modular inclusion construction leads to a generalized chiral theory whose longitudinal interval-associated net $\{\mathcal{A}(I)\}_{I \subset \mathrm{R}}$ (where $\stackrel{\circ}{\mathrm{R}}$ denotes the compactified lightray) should be pictured as the operator algebra of a d-2 dimensional strip of width $I$ in longitudinal direction. We want to create a transversal net structure i.e. construct algebras $\mathcal{A}\left(\mathcal{S}\left(\mathcal{O}_{\perp}\right)\right)$ belonging to the two-sided longitudinal extension of a d-2 dimensional compact region on the edge of the horizon (or of the wedge $W$ ). If we would have a net of algebras associated with compact regions $\mathcal{O}_{L F} \subset L F$ on the d-1 dimensional lightfront we could form these strip algebras by the weak closure of algebras $A\left(\mathcal{O}_{L F}\right)$ in the usual manner

$$
\mathcal{A}\left(\mathcal{S}\left(\mathcal{O}_{\perp}\right)\right)=\overline{\bigcup_{O_{L F} \subset \mathcal{S}\left(\mathcal{O}_{\perp}\right)} \mathcal{A}\left(\mathcal{O}_{L F}\right)}
$$

Such a net structure can indeed be obtained via applying Wigner's little group transformations which preserves the direction of the horizontal lightray. In fact the idea was first used in a joint paper with Wiesbrock 25] (chiral "scanning") in order to enrich the standard modular inclusions by forming intersections ("modular intersections") of the original $W$ with L-tilted wedges $W_{i}$ which share the upper defining lightray and as a consequence also the upper horizon. Projected into the lightfront the translation part of the Wigner little group is represented by a Galilei-transformation $G_{v}$

$$
\mathbf{x}_{\perp} \rightarrow \mathbf{x}_{\perp}+\mathbf{v} x_{+}, x_{+} \rightarrow x_{+}
$$

\footnotetext{
${ }^{8}$ In the case of free-field generated algebras the structure of the lightfront algebras can be simply obtained by restricting the underlying free fields to the lightfront [20], and the use of the modular inclusion techniques and the lightlike Wigner little group would be unnecessary.

${ }^{9}$ In fact one can show directly that the transverse symmetry wrecks the split property in the lightray direction, i.e the fuzzy localized intermediate type I factors cannot exist.
} 
The d-2 dimensional strip algebra $\mathcal{A}(I)$ in the direction of the transverse bifurcation edge is transformed into an inclined position within the lightfront. The compact intersections of the original with the transformed strip leads to local algebras (which according to the condition of the previous section are nontrivial algebras)

$$
\mathcal{A}\left(\mathcal{S}\left(\mathcal{O}_{\perp}\right)\right) \cap \mathcal{A}\left(G_{v}\left(\mathcal{S}\left(\mathcal{O}_{\perp}\right)\right)\right)
$$

which are the desired building blocks for the compact $\mathcal{O}_{L F}$ localization region used for defining the net of operator algebras $\mathcal{A}\left(\mathcal{O}_{L F}\right)$ on the lightfront from which in turn the longitudinal strip algebras (9) may be constructed. Following Driessler we now show that the foliation of the lightfront algebra $\mathcal{A}(L F)$ into nonoverlapping commuting longitudinal strip algebras

$$
\begin{aligned}
\mathcal{A} & =\mathcal{A}(L F)=\overline{\bigcup_{i} \mathcal{A}\left(\mathcal{S}\left(\mathcal{O}_{\perp}^{(i)}\right)\right)} \\
L F & =\bigcup_{i} \mathcal{S}\left(\mathcal{O}_{\perp}^{(i)}\right), \mathcal{O}_{\perp}^{(i)} \cap \mathcal{O}_{\perp}^{(j)}=\emptyset, i \neq j
\end{aligned}
$$

has a tensor product structure i.e. fulfills the strongest form of statistical independence which quantum physics is able to offer.

Theorem 4 The transverse foliation of the lightfront algebra is a tensor-product factorization

$$
\begin{aligned}
\mathcal{A}(L F) & =\bar{\otimes}_{i} \mathcal{A}\left(\mathcal{S}\left(\mathcal{O}_{\perp}^{(i)}\right)\right) \\
H & =\bar{\otimes}_{i} H_{i}, \Omega=\bar{\otimes}_{i} \Omega_{i} \\
H_{i} & =\overline{\left.\mathcal{A}\left(\mathcal{S}\left(\mathcal{O}_{\perp}^{(i)}\right)\right)\right) \Omega}
\end{aligned}
$$

Proof. The proof is based on the existence of a transverse "get away" shift, i.e. by translating the strip transversely outside itself we obtain according to spacelike local commutativity two commuting strip algebras and the rather surprising statement that there exists no transverse vacuum polarization. According to an old result of [27] one knows that each such algebra has a (lightlike) translation-invariant center and the lightlike translation acts as an inner automorphism. We use the projector onto the subspace which the strip algebra generates cyclically from the vacuum. Now look at the analytic properties associated with the following relation between an operator $A \in \mathcal{A}\left(\mathcal{S}\left(\mathcal{O}_{\perp}\right)\right)$ and another one from the commutant $A^{\prime} \in \mathcal{A}\left(\mathcal{S}\left(\mathcal{O}_{\perp}\right)\right)^{\prime}$

$$
\left\langle 0\left|A U_{e_{+}}(a) A^{\prime}\right| 0\right\rangle=\left\langle 0\left|A^{\prime} U_{e_{+}}^{*}(a) A\right| 0\right\rangle
$$

But using the analyticity due to the positivity of the generator, we obtain via the Liouville theorem the constancy in $a$ and hence

$$
\left\langle 0\left|A A^{\prime}\right| 0\right\rangle=\langle 0|A| 0\rangle\left\langle 0\left|A^{\prime}\right| 0\right\rangle
$$

which together with the cyclicity condition of the previous section leads to a type $I_{\infty}$ tensor factorization, as well as the stronger statement that the vacuum has no entanglement with respect to the inside-outside tensor factorization. Recursive application together with the fact that (8) $\mathcal{A}(L F)=\mathcal{A}=\mathcal{B}(H)$ yields the tensor factorization and absence of vacuum entanglement in the tiling of the lightfront by longitudinally directed strips i.e. the equations (13). The one-sided strip algebras $\mathcal{A}\left(\mathcal{S}_{+}\left(\mathcal{O}_{\perp}\right)\right)$ on the horizon $L F_{+}$ are easily shown to be type hyperfinite type $I I I_{1}$ algebras within the respective tensor factors so that $\mathcal{A}\left(\mathcal{S}\left(\mathcal{O}_{\perp}\right)\right)=\mathcal{A}\left(\mathcal{S}_{-}\left(\mathcal{O}_{\perp}\right)\right) \vee \mathcal{A}\left(\mathcal{S}_{+}\left(\mathcal{O}_{\perp}\right)\right)$. Since the argument is the same as that for wedges, it will not be repeated here [12].

This state of affairs requires some comments. In local quantum physics one encounters several situations in which the notions of "causal disjoint" becomes synonymous with "nonoverlapping". For standard chiral theories on $S^{1}$ this is well-known, but it also holds for conformal observables living in the DiracWeyl compactified Minkowski spacetime if "nonoverlapping" is interpreted as "nonoverlapping of the lightlike prolongation" (Huygens principle) 28] 29]. Via the CQFT-AdS isomorphism [6] this simplified causality picture is inherited by observables in anti deSitter spacetime. But none of these cases has such 
far-reaching factorization properties as the local quantum physics on the lightfront and (see next section) lightcones. This is why the metaphoric sounding terminology " return of QM" was used in the title of this section.

The usefulness of auxiliary constructs and associated (holographic) reprocessing of degrees of freedom depends very much on how the degrees of freedom "align" themselves after they received their new "spacetime-indexing". In this respect the holographic reprocessing onto causal horizons (for the rotational case see next section) is extraordinarily rich because it preempts the transversal factorization structure which is necessary for area proportionality of vacuum-split caused entanglement entropy (i.e. entropy per unit transversal volume).

In order to convert this multiplicative transverse composition law of lightfront degrees of freedom into an additive area law we should search for an appropriate notion of entropy in which the vacuum turns into a temperature state. This certainly does not happen relative to the full quantum mechanical strip algebras $\mathcal{A}\left(\mathcal{S}\left(\mathcal{O}_{\perp}\right)\right)$ relative to which the vacuum remains a pure state. The situation changes if by cutting the strip into two pieces we restrict the vacuum to the horizon component $\mathcal{A}\left(\mathcal{S}_{+}\left(\mathcal{O}_{\perp}\right)\right)$. In this case the vacuum state turns into a temperature state at the Hawking temperature $T=2 \pi$ (in appropriate units) but since this algebra is easily shown to be a hyperfinite type $\mathrm{III}_{1}$ factor (for the same reason as the wedge algebras 12 ) this is bad for the definition of an entropy since such algebras simply do not permit to associate an entropy to any state. The reason for this are the uncontrollable vacuum fluctuations at the boundary, a phenomenon well-known from the divergencies in the definition of partial Noether charges involving a sharp spatial cutoff as mentioned in the previous section. As in that case the choice of smearing functions which have the constant value one in the desired localization region of the partial charge and zero outside a slightly bigger region (thus leaving a "collar" inside which the fluctuation can be controlled) the split property achieves something similar for algebras. Given two hyperfinite type III $_{1}$ algebras, one sharply localized in a causally closed double cone $\mathcal{O}$ and the other in $\mathcal{O}+\delta$ (where $\delta$ is the collar size). If there exists an intermediate quantum mechanical algebra $\mathcal{N}$ the inclusion is called the "split" 10. In this case one achieves a control of vacuum polarizations through the use of the quantum mechanical type I factor $\mathcal{N}$ between $\mathcal{A}(\mathcal{O})$ and $\mathcal{A}(\mathcal{O}+\delta)$

$$
\begin{aligned}
\mathcal{A}(\mathcal{O}) & \subset \mathcal{N} \subset \mathcal{A}(\mathcal{O}+\delta) \\
\mathcal{A} & =B(H)=\mathcal{N} \bar{\otimes} \mathcal{N}^{\prime} \\
H & =H_{\text {in }} \bar{\otimes} H_{\text {out }}
\end{aligned}
$$

The last two lines express the tensor factorization of the algebra into "inside and outside degrees of freedom i.e. those contained in $\mathcal{A}(\mathcal{O})$ and those in $\mathcal{A}(\mathcal{O}+\delta)^{\prime}$ i.e. localized "outside" (meaning in the relativistic setting: causally disjoint from) $\mathcal{O}+\delta$. This is the best analogy of the "inside/outside" tensor factorization of (second quantized) quantum mechanics which the presence of vacuum polarization and the causal shadow property permits. It has turned out that contrary to the aforementioned Noether charge analogy which depends on the form of the smearing function in the collar region, the intermediate fuzzylocalized (between $\mathcal{O}$ and $\mathcal{O}+\delta$ ) algebra $\mathcal{N}$ is canonically (more precisely functorially, [30]) related to the given pair. The restriction of the vacuum to $\mathcal{N}$ is not only a thermal state at a Hawking temperature, but it also permits a conventional density matrix description

$$
\begin{aligned}
\left.\omega\right|_{\text {res }} & \rightarrow \rho, \rho>0, \operatorname{tr} \rho=1 \\
S_{\text {ent }} & =-\operatorname{tr} \rho \ln \rho
\end{aligned}
$$

which is the prerequisite for defining a von Neumann entropy. Clearly such an entropy would have a geometric localization aspect (i.e. it would represent an entropy associated with the localization-caused temperature) as well as a quantum interpretation in form of a reduction of the vacuum entanglement $\left(\Psi_{i}, \Phi_{j}\right.$ basisvectors in $\left.H_{\text {inn }}, H_{\text {out }}\right)$ to the subsystem $\mathcal{N}$.

$$
\begin{aligned}
\Omega & =\sum_{i, j} a_{i j} \Psi_{i} \bar{\otimes} \Phi_{j} \\
\rho & =\left(\rho_{i k}\right) \text { in } \Psi-\text { basis of } H_{i n n} \\
\rho_{i k} & =\sum_{j} a_{i j} a_{k j}^{*}
\end{aligned}
$$


In order to have a finite entropy the density matrix $\rho$ should not have too many eigenvalues near zero.

The clarity of these concepts and their mathematical formulations stands in contrast to the difficulties one encounters in attempts to calculate or estimate $\rho$ and the associated entropy along these lines. Even in the simplest setting of chiral theories this has not been achieved. There is another definition of entropy which uses the same physical intuition but whose mathematical relation to (18) is not known. This is Araki's relative entropy between two states on the same algebra in our case between the vacuum state $\omega$ and the product vacuum $\omega_{p}$ [31] on $\mathcal{A}(\mathcal{O}) \vee \mathcal{A}(\mathcal{O}+\delta)^{\prime}$. Kosaki found a beautiful variational representation of this relative entropy which avoids the use of modular theory and uses only the two states. Again this entropy unfortunately resisted up to now calculational attempts for the problem at hand [31]. There are other calculational more accessible formulas for localization entropy which are less canonical and (analog to the testfunction dependence of the above partial charge) dependent on how one realizes the two states. Intuitively one expects that the leading behavior for $\delta \rightarrow 0$ is the same, but a rigorous proof for this is still missing. One such family of formulas uses the connection between tensor product doubling and the introduction of doublet fields [32]. In this doublet description the unitary operators $U(f)$ which implements the split isomorphism and transforms the vacuum into its split version can be written in terms of a Noether current $j$ defined by the doublet integrated with testfunctions $U(f)=e^{i j(f)}$ subject to certain support requirements (in analogy to the partial charge, the $f$ is chosen such that in $\mathcal{O}$ one has $f=1$, whereas in the causal disjoint $f$ vanishes). For the split algebra generated by a free chiral Fermion this suggests a linear vanishing with decreasing collar size $\delta \rightarrow 0$ [5]. Since the split situation in this limit is known to become inequivalent to the vacuum representation of the limiting theory $\mathcal{A}(\mathcal{O}) \vee \mathcal{A}\left(\mathcal{O}^{\prime}\right)$, this vanishing property is inherited by all vectors which are cyclically generated by applying operators from the algebra to the doubled vacuum $\Omega \bar{\otimes} \Omega$ and its $U(f)$ transform. With all overlaps approaching zero linearly with vanishing distance $\delta$, one naturally expects the linear vanishing of the $a_{i k}$ coefficients in the decomposition of the vacuum in the $U(f)$ rotated basis (18) which then would lead to a logarithmic increase of the associated entropy.

It is very gratifying that the holographic lightfront projection leads to generalized chiral theories where such calculational techniques may be applied. However the simplest split of longitudinal separation on one fixed lightfront which one would use in standard chiral theories only works for massive theories in $\mathrm{d}=1+1$ when there is no chiral extension. Whenever the horizon has a transverse extension i.e. for $d \geq 1+2$ there are too many degrees of freedom which destroy the split property 10 (even though the original theory before the holographic projection has the split property) as it was already evident from our previous remark that a lightcone restricted free field is effectively a generalized free field with a continuous mass distribution depending on $\left|p_{\perp}\right|$ (i.e. there exists no density matrix description for the restricted vacuum state without the split property). As soon as we work on two lightlike separated lightfronts and continue the strip after crossing the edge on the second lightfront (in which case the distance becomes spacelike instead of lightlike) we recover the split property. This makes the application of the above Noether idea for the construction of $U(f)$ somewhat more involved but a straightforward geometric argument shows that the transverse factorization formula (13i) is recovered in the limit $\delta \rightarrow 0$ when both lightfronts coalesce. Such free field calculation combined with the fact the scale dimensions of the generalized chiral theories on the lightfront only involve canonical (halfinteger spin) scale dimensions strongly suggest the following picture for the localization entropy of the strip algebras on the horizon and the nature of the generators of the generalized chiral algebras:

- The vacuum polarization strength in the limit $\delta \rightarrow 0$ is universal (logarithmic)

- The leading coefficient for $\delta \rightarrow 0$ is proportional to the $\mathrm{d}-2$ dimensional transverse width of the strip (the area law) and the remaining factor is "almost" kinematical (depends maximally on lightfront universality classes). This suggests to split off the universally diverging factor, which originates from the ubiquitous presence of (lightlike) vacuum polarization as well as the area factor, which is the consequence of the degeneracy from

- the transverse translation symmetry. The finite numerical coefficient which remains (which can be computed from a chiral theory on the lightray) is the looked for (relative) area density of localization entropy. It retains the right additive behavior for statistically independent subsystems

\footnotetext{
${ }^{10}$ This point was overlooked in a previous version of this work.
} 
on the horizon, but is only determined up to a common (quantum matter independent) factor. The remaining normalization problem requires the derivation of basic laws of thermodynamics for this new type of horizon-associated entropy, an issue which is outside the scope of this paper.

- The generating fields of the generalized chiral lightfront algebra are of the form $\left\{\varphi\left(x_{+}, f\right) \mid f \in L^{2}\left(R^{d-2}\right)\right\}$ where the $\varphi$ have (half)integer scale dimensions and commute for orthogonal $f$.

These properties, if they stand up to closer scrutiny, reinforce the quantum mechanical transverse area structure of the above theorem. In particular the second statement which solves the problem of converting the multiplicative area structure of lightfront degrees of freedom into an additive area law would be the perfect quantum match for the classical Bekenstein area law. In fact the derivation of area proportionality for black holes entropy via inferring thermodynamical fundamental laws on Hawking's quantum thermal observations would be explained in terms of a totally generic local quantum physical property which in itself does not require the presence of special curved spacetime backgrounds.

This extremely useful role of lightfront holography in the quest for a generic quantum Bekenstein area law is somewhat at odds with recent speculations about the role of "branes" in local quantum physics. In that case the causal shadow extensions into the ambient spacetime prevent their interpretation as independent physical objects. This raises the question whether brane concepts together with the closely related Kaluza-Klein reduction and many other string-theory supported ideas can be consistent with the causal shadow property and the control of vacuum fluctuations for small additional spatial dimensions outside the quasiclassical approximation. For physicists who study semiclassical aspects of branes and also believe that paradoxes and contradictions contain the enigmatic force for progress this is an interesting situation.

It is worthwhile to point out that the use of algebraic lightfront description goes far beyond its connection with the Bekenstein area law. It constitutes a new tool of local quantum physics which offers all the advantages of the old equal time canonical formalism without suffering from its short distance limitations. Whereas the canonical formalism required the finiteness of the wave function renormalization constants $Z$ (finiteness of the integral over the Kallen-Lehmann spectral functions) and therefore excludes all properly renormalizable models, the algebraic holography based on modular inclusion avoids the illdefined restriction of fields to space- or lightlike- subspaces. Furthermore the chiral theories obtained by this construction are, as already mentioned, of an extreme kinematical and universal kind. This is because the modular inclusion method encodes the spacelike Boson/Fermi structure in the original formulation into (half)integer dilatation spectrum on the horizon. In fact the lightfront algebra can be described in terms of canonical (half)integer dimension generating fields [33], but they have no obvious connection to the pointlike fields which may have generated the original algebras; the latter have in general anomalous short distance dimension. The scale spectrum of the kinematical chiral theory on the other hand results from the statistics and is not related to the anomalous short distance scale spectrum of the original theory. Although both the scaling short distance limit and the lightfront holographic limit exhibit conformal symmetry, the lightfront theory is much more "universal" than the short distance universality classes used in the description of critical phenomena. In addition the latter allow no mathematically controllable (since they live in different Hilbert spaces) return to the original theory whereas the lightfront holography reveals its massive origin upon application of the Poincaré covariances. In short, the algebraic lightfront formalism seems to be the long looked for dynamical "El Dorado": a (almost) universal kinematical operator algebra on the lightfront (see the above conjecture about the structure of its generating fields) which becomes reprocessed into the rich world of quantum field theoretic models by differently acting automorphisms. As a result of their short distance limitations neither the canonical formalism nor the closely related Euclidean action method could play this role, although they both served (and still serve) as useful artistic catalyzers of thoughts about particle physics 1 (see also remarks in the last section). But in order to convert this kinematical El Dorado into a new powerful constructive tool of QFT one needs to get a much better conceptual understanding and mathematical control of fuzzy acting (auto)morphisms.

What one should do is to rearrange degrees of freedom (as it already was done in the holographic projection onto the lightfront) rather than throw away some of them. Precisely this is achieved by the splitting method which consists in creating a small bit finite distance between them. Here the analogy to

\footnotetext{
${ }^{11}$ The correctly renormalized physical answers only fulfill Einstein causality but are neither canonical nor Feynman-Kac representable.
} 
the inside/outside nonrelativistic quantization box (in the multiplicative formulation of $2^{\text {nd }}$ quantization in order to make the analogy closer) breaks down, because by removing some spatial localization region (for all times) one is really dumping degrees of freedom. A closer examination of the QFT situation reveals is that what splitting does is to avoid uncontrollable divergencies which are invariably created by vacuum fluctuations if they are forced to take place directly in a surface (or in a point in the chiral case). Both localizations regions in this way have a fuzzy extension into the "collar" and this fuzzy splitting is only a rearrangement and does not involve throwing away degrees of freedom.

\section{Comment on rotational horizons}

Let us now consider the more difficult task of a rotational symmetric double cone and its noncompact causal disjoint. One immediately notices some analogies to the previous planar bifurcated causal horizon. The characteristic causal shadow of the lower lightcone horizon $h_{-}(C)$ of the unit double cone $C$ (placed symmetric around the origin) agrees with the double cone and its complement on the mantle of the future lightcone $h_{+}\left(V_{+}\right)=h\left(V_{+}\right) \backslash h_{-}(C)$ is the rotational analog of the upper horizon of the Rindler wedge. Instead of the planar longitudinal strips we now consider strips into radial directions with a fixed space angle opening which start at the rotational symmetric edge of bifurcation and extends to lightlike infinity. In this way the horizon $h_{+}\left(V_{+}\right)$may be partitioned into radial strips $\mathcal{S}_{i}$ which cast no causal shadow and whose associated algebras mutually commute. This is the prerequisite for an angular tensor product foliation

$$
\begin{aligned}
& \overline{\mathcal{A}\left(h_{+}\left(V_{+}\right)\right) \Omega}=H \\
& H=\overline{\bigotimes_{i}} H_{i}, H_{i}=\overline{\mathcal{A}\left(\mathcal{S}_{i}\right) \Omega}
\end{aligned}
$$

The crucial question is whether there exists a substitute for a lightlike translation with positive generator whose analytic properties implies the transverse (now in the rotational sense) factorization of the vacuum into entanglement-free strip vacua in analogy to the theorem in the previous section.

It is precisely at this point where the analogy becomes somewhat opaque. Part of the difficulty results from the fact that the rotational causal horizon does not come with a Killing vector field (analogous to event horizons in curved spacetime which are not associated with Killing symmetries), except in case the QFT is massless. In that case the Ad-action of the modular unitary and the Tomita involution $J$ of the unit double cone corresponds to the following point transformations (the origin is in the center of the unit double cone 10 )

$$
\begin{gathered}
A d \Delta^{i \frac{s}{2 \pi}}: x_{ \pm}(s)=\frac{(\cosh s) x_{ \pm}+(\sinh s)}{(\sinh s) x_{ \pm}+(\cosh s)}, x_{ \pm} \equiv x^{0} \pm|\vec{x}| \\
J: x^{i} \rightarrow-\frac{x^{i}}{x^{2}}, x^{0} \rightarrow \frac{x^{0}}{x^{2}}, \quad-1<x_{ \pm}<1
\end{gathered}
$$

Although an explicit description of the corresponding fuzzy modular transformations in the massive case is presently not possible the modular objects become geometric in the holographic projection in which the double cone is projected onto its lower lightcone mantle and the spacelike disjoint projects on the infinite complementary outside part of the mantle.

Remark 5 The restriction of the double-cone localized free massive field to the lower horizon (i.e. to the mantle of that part of the forward lightcone whose causal shadow is the double cone) follows the method used for restricting free field operators to $L F_{+}[34]$ : the limit $x_{-}=x^{0}-|\vec{x}| \rightarrow 0$ in the plane wave factor (with the origin at the lower apex of the double cone) is compensated by an $\ln x_{-}$-increase in the spatial rapidity $\chi$; the creation/annihilation operators remain unaffected. The resulting free field restriction is that of a massless field (the mass only remains as a scale factor in the exponential), however the physical mass is re-activated by acting with Poincaré transformations on the lightcone restriction.

\footnotetext{
${ }^{12}$ The conjecture that these transformation correspond to double cone (and its causal disjoint) support preserving test function transformations of the pseudo-differential kind [25] still stands.
} 
It is believed that the holographic lightcone projection of all massive theories admits this geometric modular group action

$$
\begin{gathered}
A d \Delta^{i \frac{s}{2 \pi}}: x_{+}(s)=\frac{(\cosh s) x_{+}+(\sinh s)}{(\sinh s) x_{+}+(\cosh s)} \\
x_{-}=0, \quad x_{+} \equiv x^{0}+|\vec{x}|,\left|x_{+}\right| \leq 1
\end{gathered}
$$

and on the holographic projection of its causal disjoint $x_{-}=0, x_{+} \geq 1$. This leads to the interesting conjecture that the theorem about modular inclusion of the previous section may have a partially geometric counterpart in which two fuzzy modular groups 13 may nevertheless lead to a geometric Borchers pair (positive lightlike translation, dilation) on the lightcone horizon. Further work is necessary to clarify the situations of causal (event) horizons without Killing symmetries.

\section{Concluding remarks}

My use of the adjective "paradigmatic" in the title requires more justification.

In most uses of methods of AQFT there was always a way to see things (perhaps not in the most elegant fashion) in terms of field-coordinatizations. In the case at hand, as a result of severe short distance limitations which (except for free fields) rule out to define holographic lightfront projections by restricting pointlike fields, this is not possible.

The main point in the present work was to establish a characteristic property of a new algebraic approach to "holographic lightfront projection" (including a precise definition), namely its perfect transverse quantum mechanical aspect. According the best of my knowledge this is the only known case where Galilei-invariant vacuum-polarization-free quantum mechanics appears within QFT as a rigorous structural property i.e. without any nonrelativistic approximation $v<<c$.

The prerequisite for this extraordinary phenomenon is the spacetime arrangement suffered by local quantum physical degrees of freedom on non-globally hyperbolic manifolds. Situations as the AdS-CQFT correspondence [35] (also often referred to as AdS-CQFT holography) (in which the local form of causal propagation is still maintained ${ }^{14}$, are not radical enough for obtaining such a situation. One really needs the absence of causal shadows as encountered on causal (event) horizons where only seminifinite regions which extend to lightlike infinity are capable of casting causal shadows into the ambient spacetime. The operator algebras associated with such seminifinite regions are the only ones which geometrically communicate between the lightfront and its ambient spacetime.

This use of change of spacetime indexing as a tool of structural investigation in QFT links up very nicely with a recent stunning discovery about the true nature of QFT [9]. Whereas prior to that observation the curved spacetime aspect were always considered as part of the specification of the model, the new view of QFT is that of a functor between the categories of globally hyperbolic Lorentz signature (sub)manifolds (with the arrows being isometric embedding) and that of operator algebras (with the arrows being morphisms). In other words the curved spacetime aspect is not an additional property imposed on a QFT but belongs to the very definition of what constitutes a QFT. In order to illustrate this paradigmatic change of thinking about QFT let me be somewhat metaphoric in my analogies and compare the abstract form of QFT with a kind of unstructured mold of degrees of freedom (being structured in different ways by different space-time indexing ${ }^{15}$ ) similar to stem-cells and their versatile use for the generation of different biological tissue. The spacetime acting as agents for structuring the degree of freedoms by forcing them to align in a certain way. If anything, the present modular supported holography deepens this surprising new view in that it permits to change the spacetime indexing in an even more

\footnotetext{
${ }^{13}$ It has been conjectured that the action of fuzzy modular actions on testfunctions should be described by a special type of pseudo-differential operators which leaves their given $\mathcal{O}$-support and its causal disjoint invariant [39] but unforunately no progress has been obtained on this point.

${ }^{14}$ In a recent paper 37 it was shown that the string-theory induced duality relation of AdS with CQFT is just an alternative formal reformulation of the field theoretic restriction at the conformal boundary at infinity (which permits a rigorous algebraic presentation 36 ).

${ }^{15}$ An illustration would be the abstract Weyl algebra as an operator-valued functor on abstract Hilbert spaces versus the concrete labeling by identifying the abstract space as a concrete function space and using the localized subspaces of the latter to generate a concrete net structure on the former.
} 
(not generally understood) radical way by allowing lower dimensional sets. It would be nice if algebraic holographic projections onto horizons could be viewed as a generalization of spacetime reprocessing to situations in which there are no connecting local diffeomorphisms as in [9].

Extrapolating from properties of interaction-free lightfront holography in the present work, one expects the existence of a relative area density of localization entropy associated with the horizon. There are two prerequisites which would insure the validity of an area law as the local quantum counterpart of the classical Bekenstein law for black hole entropy. The "split entropy" of the vacuum restricted to the algebra of a split interval in a chiral theory associated to the lightfront should increase with the splitting distance $\varepsilon \rightarrow 0$ in a universal way so that the ratio between two models with different matter content but the same geometric split situation stays finite. For the normalization of such a relative entropy one needs a second prerequisite which is the validity of a fundamental thermodynamic law which relates the entropy to other already normalized quantities.

In contrast to this envisaged generic area behavior, there have been derivations of area laws for some kind of horizon-affiliated entropy (whose precise local quantum physical interpretation is not clear) in models of string theory [38] or by imposing a classical Virasoro structure on horizons and quantizing [40], or last not least by rewriting canonical classical GR variables into loop-like variables [41]. Although the conditions of their derivation seems to be too special in order to account for the apparently universal Bekenstein area law (unless one believes in the TOE doctrine for those models), they are not necessarily contradicting the present lightfront holography mechanism. It is conceivable that a local quantum phenomenon, which is generic in the algebraic setting but at the same time not easily accessible in the more geometric standard formulation of QFT, may well present itself in special geometric situations in a more manifest fashion.

The present view de-emphasizes somewhat the importance of black holes as a direct entrance ticket into QG. But not completely, since there is the very interesting message of an apparent very deep connection of thermal physics with geometry at the place where one wants to see new quantum gravity degrees of freedom. This has already been foreshadowed by recent results on the construction of external and internal symmetries and spacetime geometry from the relative position of operator algebras [17] [2] [7], and in particular the emergence of infinite dimensional fuzzy analogs of diffeomorphism groups (including the Poincaré and conformal diffeomorphisms) from modular inclusions and intersections of algebras point into the same direction [43].

Acknowledgment: I am thankful to Detlev Buchholz for directing my attention to the almost forgotten early work of W. Driessler. I also acknowledge critical remarks of K.-H. Rehren which led to some modifications of the first version.

\section{Appendix: Lightfront restriction}

We start with a $\mathrm{d}=1+1$ massive free field in the momentum space rapidity description

$$
\begin{aligned}
A(x) & =\frac{1}{\sqrt{2 \pi}} \int\left(e^{-i p x} a(\theta)+e^{i p x} a^{*}(\theta)\right) d \theta \\
p & =m(\operatorname{ch} \theta, \operatorname{sh} \theta)
\end{aligned}
$$

The positive part $x_{+} \equiv t+x>0$ of the light ray $x_{-}=t-x=0$ which is the upper horizon of the wedge $t^{2}-x^{2}<0, x>0$ is approached by taking the $r \rightarrow 0, \chi=\hat{\chi}-\ln \frac{r}{r_{0}} \rightarrow \hat{\chi}+\infty$ in the x-space rapidity parametrization

$$
\begin{aligned}
& x=r(\operatorname{sh} \chi, \operatorname{ch} \chi), x \rightarrow\left(x_{-}=0, x_{+} \geq 0, \text { finite }\right) \\
& A\left(x_{+}, x_{-} \rightarrow 0\right) \equiv A_{L F}\left(x_{+}\right)=\frac{1}{\sqrt{2 \pi}} \int\left(e^{-i p_{-} x_{+}} a(\theta)+e^{i p_{-} x_{+}} a^{*}(\theta)\right) d \theta \\
& =\frac{1}{\sqrt{2 \pi}} \int\left(e^{-i p_{-} x_{+}} a(p)+e^{i p_{-} x_{+}} a^{*}(p)\right) \frac{d p}{|p|}
\end{aligned}
$$

where the last formula serves to make manifest that the limiting $A_{L F}\left(x_{+}\right)$field is a chiral conformal (gapless $P_{-}$spectrum) field; the mass $m$ in the exponent $p_{-} x_{+}=m r_{0} e^{\theta} e^{-\hat{\chi}}$ is a dimension preserving 
parameter which has lost its physical significance of a mass gap; the physical mass is related to the gap in the $P_{-} \cdot P_{+}$spectrum of the ambient $\mathrm{d}=1+1$ theory. Note that unlike the scaling limit, the lightfront only effects the numerical factors and not the Fock space operators $a^{\#}(\theta)$.

In this formal argument using pointlike fields one has to keep in mind that a free massless $\operatorname{dim} A=$ 0 scalar field is only defined on a restricted space of localized test functions. This restriction arises automatically if one performs the limit in a more careful way including smearing with wedge-supported test functions. With supp $\tilde{f} \in W, \tilde{f}$ real, one obtains

$$
\begin{aligned}
& \int A\left(x_{+}, x_{-}\right) \tilde{f}(x) d^{2} x=\int_{C} a(\theta) f(\theta)=\int A_{L F}\left(x_{+}\right) \tilde{g}\left(x_{+}\right) d x_{+}, \tilde{g} \text { real } \\
& \tilde{f}(x)=\int_{C} e^{i p(\theta) x} f(\theta) d \theta, \tilde{g}\left(x_{+}\right)=\int e^{i p x_{+}} g(p) \frac{d p}{|p|}=\int_{C} f(\theta) e^{i p_{-}(\theta) x_{+}} d \theta
\end{aligned}
$$

These formulas, in which a contour $C$ appears, require some explanation. The on-shell character of free fields restricts the Fourier transformed test function $f(p)$ to their mass shell values with the backward mass shell corresponding to the rapidity on the real line shifted downward by $-i \pi$

$$
\left.f(p)\right|_{p^{2}=m^{2}}=\left\{\begin{array}{c}
f(\theta), p_{0}>0 \\
f(\theta-i \pi), . p_{0}<0
\end{array}\right.
$$

and the wedge support property is equivalent to the analyticity of $f(z)$ in the strip $-i \pi<\operatorname{Im} z<0$. The integration path $C$ consists of the upper and lower rim of this strip and hence corresponds to the negative/positive frequency part of the Fourier transform. The vanishing of the massless test function $g(p)$ is nothing but the statement that the wave function $f(\theta)$ considered as a zero mass shell restriction of a massless test function $g(p)$ must have the vanishing property at $p=0$ since this corresponds to the value $\theta=-\infty$ of the square integrable $f(\theta)$

$$
\begin{gathered}
\left.f(p)\right|_{p^{2}=m^{2}, p_{0}>0} \frac{d p}{\sqrt{p^{2}+m^{2}}}=f(\theta) d \theta \equiv g(\theta) d \theta=\left.g(p)\right|_{p^{2}=0, p_{0}>0} \frac{d p}{|p|} \\
\curvearrowright g(p=0)=0, \text { or } \int \tilde{g}\left(x_{+}\right) d x_{+}=0
\end{gathered}
$$

with a similar formula for negative $p_{0}$ and the corresponding $\theta$-values at the lower rim. Note that this argument excludes the "naive" (unrestricted) $\operatorname{dim} A=0$ zero mass free field which would violate Huygens principle, require indefinite metric and violate cluster properties (and hence contradict the main theorem in section 3).

The derivation of these formulas for $d>1+1$ becomes word for word the same if one introduces the effective mass $m_{\text {eff }}=\sqrt{m^{2}+p_{\perp}^{2}}$ where $p_{\perp}$ is transverse to the lightray momentum. In that case one should do the calculation on the basis of longitudinal/transverse product test functions $f(p)=$ $f_{l}\left(p_{l}\right) f_{t}\left(p_{\perp}\right)$ and extend linearly after the lightfront limit has been performed. The $f_{l}$ passes to a massless $g_{l}$ just as in the $\mathrm{d}=1+1$ case. In terms of the two-point function the result is

$$
\begin{aligned}
& \left\langle A_{L F}\left(f_{l} f_{\perp}\right) A_{L F}\left(f_{l}^{\prime} f_{\perp}^{\prime}\right)\right\rangle=\int \bar{f}_{l}\left(p_{l}\right) f^{\prime}\left(p_{l}\right) \frac{d p_{l}}{2\left|p_{l}\right|} \int \bar{f}_{\perp}\left(p_{\perp}\right) f_{\perp}^{\prime}\left(p_{\perp}\right) d^{2} p_{\perp} \\
& {\left[A_{L F}\left(x_{+}, x_{\perp}\right), A_{L F}\left(x_{+}^{\prime}, x_{\perp}^{\prime}\right)\right]=i \Delta\left(x_{+}-x_{+}^{\prime}\right)_{m=0} \delta\left(x_{\perp}-x_{\perp}^{\prime}\right)}
\end{aligned}
$$

i.e. the commutation of the transverse part is like that of Schrödinger field. In fact the analogy to QM is much stronger since the vacuum does not carry any transverse correlation at all. The total correlationless property is best seen in the algebraic transcription using the Weyl generators

$$
\begin{aligned}
\left\langle W\left(f_{l}, f_{\perp}\right) W\left(f_{l}^{\prime}, f_{\perp}^{\prime}\right)\right\rangle & =\left\langle W\left(f_{l}, f_{\perp}\right)\right\rangle\left\langle W\left(f_{l}^{\prime}, f_{\perp}^{\prime}\right)\right\rangle \text { if supp } f_{\perp} \cap \operatorname{supp} f_{\perp}^{\prime}=\emptyset \\
W\left(f_{l}, f_{\perp}\right) & =e^{i A_{L F}\left(f_{l} f_{\perp}\right)}
\end{aligned}
$$

i.e. the vacuum behaves quantum mechanically in the transverse direction. 
In the interacting case when the integral over the Kallen-Lehmann spectral function diverges $\int \rho(\kappa) d \kappa=$ $\infty$ one has to use the algebraic modular inclusion method instead of the lightfront restriction formalism.

The quantum mechanical transvers aspect of LF-QFT is corroborated by the fact that its 7-parametric symmetry group (which is a subgroup of the 10-parametric Poincaré group) contains a copy of the transvers Galilei group [5] [8].

\section{References}

[1] H. Leutwyler, J. R. Klauder and L. Streit, Nuovo Cim. 66A, (1970) 536

[2] G. 't Hooft, in Salam-Festschrift, A. Ali et al. eds., World Scientific 1993, 284

[3] L. Susskind, J. Math. Phys. 36, (1995) 6377

[4] R. M. Wald, Quantum Field Theory in Curved Spacetime and Black Hole Thermodynamics, The University of Chicago Press 1994, and references cited therein

[5] B. Schroer, Lightfront holography and area density of entropy associated with localization on wedgehorizons, and references therein, hep-th/0208113 to appear in IJMPA

[6] K.-H. Rehren, Ann. Henri Poincaré 1, (2000) 607

[7] D. Buchholz, Algebraic Quantum Field Theory: A Status Report, hep-th/00112701

[8] S. Hollands and R. Wald, Commun. Math. Phys. 223, (2001) 289

[9] R. Brunetti, K. Fredenhagen and R. Verch, The Generally Covariant Locality Principle-A New Paradigm for Local Quantum field Theory, hep-th/01122000 and references on prior work

[10] R. Haag, Local Quantum Physics, Springer Verlag (1992)

[11] B. Schroer, Uniqueness of Inverse Scattering Problem in Local Quantum Physics, hep-th/0106066

[12] H. J. Borchers, J. Math. Phys. 41, (2000) 3604

[13] H. Halvorson, Reeh-Schlieder Defeats Newton-Wigner: On alternative localization schemes in relativistic quantum field theories and references therein, quant-ph/0007060

[14] B. Schroer, Basic Quantum Theory and Measurement from the Viewpoint of Local Quantum Physics, in Trends in Quantum Mechanics, page 274, ed. H.-D.Doebner, S. T. Ali , M. Keyl and R. F. Werner, World Scientific 2000, quant-ph/9904072

[15] R. F. Streater and A. S. Wightman, PCT, Spin\&Statistics and all That, Benjamin 1964

[16] R. Haag and B. Schroer, J. Math. Phys. 3, (1962) 248

[17] H.-W. Wiesbrock, Comm. Math. Phys. 158, (1993) 537

[18] D. Guido, R. Longo and H.-W. Wiesbrock, Commun. Math. Phys. 192, (1998) 217

[19] Selected papers on Quantum Electrodynamics, edited by J. Schwinger, Dover Publications Inc. New York 1958

[20] W. Driessler, Acta Physica Austriaca 46, (1977) 163, and references therein

[21] D. Guido, R. Longo, J.E. Roberts and R. Verch, Charged sectors, spin and statistics in quantum field theory on curved spacetimes, math-ph/9906019

[22] H.J. Borchers, D. Buchholz and B. Schroer, Commun. Math. Phys. 219, (2001) 125, and prior references quoted therein.

[23] B. Schroer. Phys. Lett. B 494 (2000) 124 
[24] B. Schroer, J. Math. Phys. 41, (2000) 3801 and ealier papers of the author quoted therein

[25] B. Schroer and H.-W. Wiesbrock Rev. Math. Phys. 12, (2000) 461

[26] E. P. Wigner, Ann. Math. 40, (1939) 149

[27] H. J. Borchers, Commun. Math. Phys. 2, (1966) 49

[28] R. Brunetti, D. Guido and R. Longo, Commun. Math. Phys. 156, (1993) 201

[29] B. Schroer, Phys. Lett. B 506, (2001) 337

[30] S. Doplicher and R. Longo, Invent. math. 75, (1984) 493

[31] H. Narnhofer, in The State of Matter, ed. by M. Aizenman and H. Araki (Wold-Scientific, Singapore) 1994

[32] C. D'Antoni, S. Doplicher, K. Fredenhagen and R. Longo, Commun. Math. Phys.110, (1987) 325

[33] M. Jörss, Lett. Math. Phys. 38, (1996) 252

[34] B. Schroer, Lightfront Formalism versus HolographyECChiral Scanning, hep-th/0108203

[35] J. M. Maldacena, Adv. Theor. Math. Phys.2, (1998) 231

[36] K.-H. Rehren, Phys. Lett.B 493, (2000) 383

[37] M. Duetsch and K.-H. Rehren, A comment on the dual field in the AdS-CFT correspondence, hepth/0204123, to appear in Lett. Math. Phys.

[38] G. Horowitz, Quantum Gravity at the Turn of the Millenium, hep-th/0011089

[39] B. Schroer and H-W Wiesbrock, Rev. Math. Phys. 12, (2000) 139

[40] S. Carlip, Nucl.Phys.Proc. Suppl.88, (2000) 10, gr-qc/9912118

[41] T. Thiemann, An Introduction to Modern Canonical Quantum General Relativity, gr-qc/0110034

[42] R. Kaehler and H.-W. Wiesbrock, JMP 42, (2000) 74

[43] L. Fassarella and B. Schroer, Phys. Lett. B 538, (2002) 415 